\title{
Crescimento somático do caranguejo-uçá Ucides cordatus (Crustacea, Brachyura, Ocypodidae) em laboratório
}

\author{
Giovana V. Lima \& Lídia M. Y. Oshiro
}

Estação de Biologia Marinha, Universidade Federal Rural do Rio de Janeiro, Rua Sereder s/nº Itacuruçá, Mangaratiba, 23880-000 Rio de Janeiro, Brasil. (giovalverde@yahoo.com.br; oshiro@ufrrj.br)

\begin{abstract}
Somatic growth of the mangrove crab Ucides cordatus (Crustacea, Brachyura, Ocypodidae) in laboratory. In crustaceans, size increase occurs only immediately after molting when the animal is in the soft-shelled condition. The growth of Ucides cordatus (Linnaeus, 1763) was observed in laboratory, through its occurrence of molt, percentual increment of size and intermolt period. The study was realized from October/2000 to March/2002 and a total of 91 crabs (15 males, 33 females and 43 juveniles) were sampled in Itacuruçá - Coroa Grande mangrove, State of Rio de Janeiro, Brazil. The animals were maintained in two types of containers: adult crabs were kept in containers of 10001 capacity, during eighteen months; while the juveniles were kept in plastic containers of 201 capacity during eight months. The crabs were maintained in flowing seawater and fed on frozen leaves of Rhizophora mangle (L.) (Rhizophoraceae) and Laguncularia racemosa (Gaertn.) (Combretaceae), twice a week. The carapace width ranged from 50.1 to 70.0 $\mathrm{mm}$ in males, 40.2 to $80.0 \mathrm{~mm}$ to females and 1.1 to $40.1 \mathrm{~mm}$ to juveniles. The survival rate of the specimens studied was of $46.7 \%$ for males, $39.4 \%$ for females after eighteen months and $67.4 \%$ for juveniles after eight months. The males and females molted up to three times during the experiment, while the juveniles molted up twice. Molts occurred between August and April, showing more frequency during spring and summer. The increment of carapace width decreased with size, with mean was $2.21 \pm 1.39 \%$ for males, $1.28 \pm 0.84 \%$ for females and $2.89 \pm 2.13 \%$ for juveniles. The relationship between the percentual increment and the carapace width can be expressed by the equation IM $=-0.0707 \mathrm{LC}+4.645\left(\mathrm{r}^{2}=0.40\right)$. The intermolt period was $191 \pm 140$ days for males, $216 \pm 76.2$ days for females and $54 \pm 1.41$ days for juveniles. The percentual increment of size was statistically significant $(\mathrm{p}<0,05)$ when compared males and females, and adults and juveniles.
\end{abstract}

KEYWORDS. Somatic growth, crab, molting, increment, intermolt period.

RESUMO. Em crustáceos, o aumento de tamanho ocorre imediatamente após a muda, quando o animal está com a carapaça mole. O crescimento de Ucides cordatus (Linnaeus, 1763) foi observado em laboratório, através da ocorrência de muda, incremento de muda e período de intermuda. O estudo foi realizado durante os meses de outubro/2000 a março/2002 e um total de 91 caranguejos (15 machos, 33 fêmeas e 43 juvenis) foi coletado no manguezal de Itacuruçá - Coroa Grande, Estado do Rio de Janeiro, Brasil. Os animais foram mantidos em dois tipos de tanques: os caranguejos adultos foram mantidos em tanques de 10001 , durante 18 meses, enquanto os juvenis foram mantidos em tanques plásticos de 201 de capacidade durante oito meses. Os animais foram mantidos em sistema aberto de circulação de água do mar e alimentados duas vezes por semana com folhas de Rhizophora mangle (L.) (Rhizophoraceae) e Laguncularia racemosa (Gaertn.) (Combretaceae). A largura da carapaça variou entre 50,1 a 70,0 mm nos machos, 40,2 a 80,0 mm entre as fêmeas e 1,1 a $40,1 \mathrm{~mm}$ entre os juvenis. A sobrevivência dos espécimes estudados foi de $46,7 \%$ entre os machos, 39,4\% entre as fêmeas após dezoito meses e $67,4 \%$ entre os juvenis, após oito meses de observação. Os machos e as fêmeas realizaram três mudas durante o experimento, enquanto os juvenis realizaram até duas mudas. As mudas ocorreram entre agosto e abril, mostrando maior freqüência durante a primavera e o verão. $\mathrm{O}$ incremento na largura da carapaça diminuiu com o tamanho do indivíduo, com média de $2,21 \pm 1,39 \%$ para os machos, $1,28 \pm 0,84 \%$ para as fêmeas e 2,89 $\pm 2,13 \%$ para os juvenis. A relação entre o incremento percentual e a largura da carapaça pode ser expresso pela equação $\mathrm{IM}=-0.0707 \mathrm{LC}+4.645\left(\mathrm{r}^{2}=0.40\right)$. O período de intermuda foi de $191 \pm 140$ dias entre os machos, $216 \pm 76,2$ dias entre as fêmeas e $54 \pm 1,41$ dia entre os juvenis. O incremento de muda foi estatisticamente significativo $(\mathrm{p}<0,05)$ quando comparados machos e fêmeas, e adultos e juvenis.

PALAVRAS-CHAVE. Crescimento somático, caranguejo, muda, incremento, período de intermuda.

Os crustáceos apresentam um padrão de crescimento diferente dos demais animais, por apresentarem vários estágios de desenvolvimento, verificando-se aumento de tamanho somente ao longo de mudas sucessivas e antes da fase de calcificação da carapaça (HANCOCK \& EDWARDS, 1967).

Segundo Lobão et al. (1996), a muda entre os crustáceos pode ser descrita como o processo de eliminação da carapaça antiga ou exoesqueleto, por um novo tegumento. Este novo tegumento, no entanto, é formado durante a pré-muda, para substituir a velha carapaça durante o processo de muda ou ecdise, permitindo, imediatamente após a liberação, o aumento de tamanho, pelo crescimento somático e pela absorção de água (HARTNOLL, 1988).

De acordo com HarTnOll (1988), o incremento de muda e o período de intermuda representam os parâmetros que determinam a taxa e o tempo de crescimento entre os crustáceos, sendo a porcentagem de aumento no tamanho do animal entre as fases de pré-muda e pós-muda caracterizada como o incremento de muda, e o tempo gasto entre duas mudas sucessivas a fase denominada período de intermuda.

Entre os diferentes crustáceos decápodos de interesse comercial, alguns estudos em relação ao processo de muda já foram realizados, como os experimentos de HarTnoll $(1982,1988)$ com Carcinus maenas (Linnaeus,1758) e outros braquiúros; TRAVIS (1954), com Panulirus argus (Latreille, 1804); Тномаs (1958), Hepper (1967) e Hewett (1974), com Homarus vulgaris $\mathrm{H}$. Milne-Edwards, 1896; AiKEN (1973), com Homarus americanus H. Milne-Edwards, 1837; SмIтH 
(1990), com Callinectes sapidus, Rathbun, 1896; (SARDÁ, 1983, 1985, 1991), com Nephrops norvegicus (Linnaeus, 1758); ReID et al. (1997) com C. maenas e SILVA \& OSHIRO (2002), com Goniopsis cruentata (Latreille, 1803).

Ucides cordatus (Linnaeus, 1763) é um caranguejo semi-terrestre pertencente à família Ocypodidae, conhecido popularmente como caranguejo-uçá ou verdadeiro, que se distribui no Oceano Atlântico Ocidental, da Florida (EUA) até o Estado de Santa Catarina (Brasil) (Melo, 1996). O caranguejo-uçá representa uma importante fonte de renda para as populações tradicionais caiçaras (NoRDI, 1994), sendo o mais importante caranguejo comercial dos manguezais brasileiros (AlcÂNTARA-Filho, 1978). De acordo com Rodrigues et al. (2000), U. cordatus se destaca ecologicamente no ecossistema devido seu importante papel no revolvimento do solo e na reciclagem de nutrientes.

Vários trabalhos têm sido realizados sobre diferentes aspectos da biologia da espécie, como o desenvolvimento larval (RodRigues \& HEBLING, 1989), reprodução (Mota Alves, 1975; Ivo \& VAsconcelos, 2000; Hattori \& Pinheiro, 2003; PinheIro et al., 2003; Dalabona et al., 2005), fisiologia (Mota Alves \& MAdeIRA, 1980; Harris \& SANTos, 2000), ecologia (Oliveira, 1946; Maia \& Penna, 1982), bioecologia (Alcântara Filho, 1978; Costa, 1979; CAStro, 1986; Branco, 1993; BlanKENSTEYN et al., 1995) e crescimento (PINHEIRo et al., 2005).

Este trabalho objetiva verificar o crescimento somático de $U$. cordatus em laboratório, averiguando o incremento de muda, período de intermuda, número de mudas e o caráter dimórfico entre os sexos.

\section{MATERIAL E MÉTODOS}

Os espécimes adultos foram coletados manualmente no manguezal de Itacuruçá - Coroa Grande, Mangaratiba, litoral sul do Estado do Rio de Janeiro, Brasil, durante o período de maré baixa por caranguejeiros locais; os juvenis foram obtidos também manualmente, vasculhando-se troncos e galhos de vegetação em decomposição no apicum do manguezal, junto a várias espécies de Uca e de Cardisoma guanhumi (Latreille, 1825). Após a captura, os animais foram transportados à Estação de Biologia Marinha da UFRRJ, onde os adultos foram mantidos durante dezoito meses (outubro/2000 a março/2002), e os juvenis por oito (julho/2001 a março/ 2002). Esse diferencial em relação ao período de observação entre juvenis e adultos ocorreu devido à obtenção dos indivíduos em diferentes datas de captura.

Foi capturado um total de 91 animais (48 adultos 15 machos e 33 fêmeas - e 43 juvenis), os quais tiveram mensurada a largura da carapaça $(\mathrm{LC}=$ ao longo da extensão da região mesobranquial) com um paquímetro de $0,01 \mathrm{~mm}$ de precisão.

Os animais foram mantidos em dois tipos de tanques: os adultos, em tanques de cimento-amianto com 1000 litros de capacidade e os juvenis em tanques plásticos "caixas de peixe" de 20 litros, que permitiram melhor visualização e manuseio dos indivíduos menores. Todos os tanques apresentaram sistema aberto de circulação da água do mar, com nível aproximado de 5 a
10 cm de coluna d'água, suficiente para permitir a emersão completa dos animais em observação. Durante o experimento, não houve utilização de qualquer tipo de substrato nos tanques, apenas pedaços de tubos de PVC de diferentes diâmetros, utilizados como abrigos pelos espécimes.

Os caranguejos foram alimentados duas vezes por semana com folhas verdes e senescentes de Rhizophora mangle (L.) (Rhizophoraceae) e Laguncularia racemosa (Gaertn.) (Combretaceae). Nenhuma folha fornecida durante a alimentação foi retirada dos tanques de observação. Diariamente os tanques foram observados e os indivíduos que sofreram muda foram devidamente identificados, tomando-se a largura da carapaça após uma semana entre os animais adultos e dois dias entre os juvenis, depois de total calcificação do novo tegumento.

$\mathrm{O}$ incremento de muda foi expresso pela porcentagem do aumento da largura da carapaça após a muda [(LC pós-muda-LC pré-muda)/LC pré-muda] x 100 e o período de intermuda determinado pelo tempo gasto, em dias, entre duas mudas consecutivas.

As relações entre o incremento de muda e o tamanho do animal e o período de intermuda e a largura da carapaça foram comparados em relação ao sexo e à maturidade, através do Teste "t" de Student, com 0,05\% de significância.

\section{RESULTADOS E DISCUSSÃO}

A largura da carapaça do caranguejo no início do experimento variou entre 50,1 a 70,0 mm nos machos, 40,2 a $80,0 \mathrm{~mm}$ nas fêmeas e entre 1,1 a $40,1 \mathrm{~mm}$ nos juvenis (Fig. 1).

A sobrevivência da espécie em laboratório foi de $51,0 \%$, embora os juvenis tenham apresentado maior sobrevivência durante os oito meses que ficaram sob observação $(67,4 \%)$. Os machos sobreviveram numa taxa de $47,6 \%$, enquanto nas fêmeas a sobrevivência foi de $39,4 \%$. A mortalidade das fêmeas com relação aos machos foi maior ao longo dos quatro primeiros meses, verificando-se, em ambos os sexos, maior estabilidade

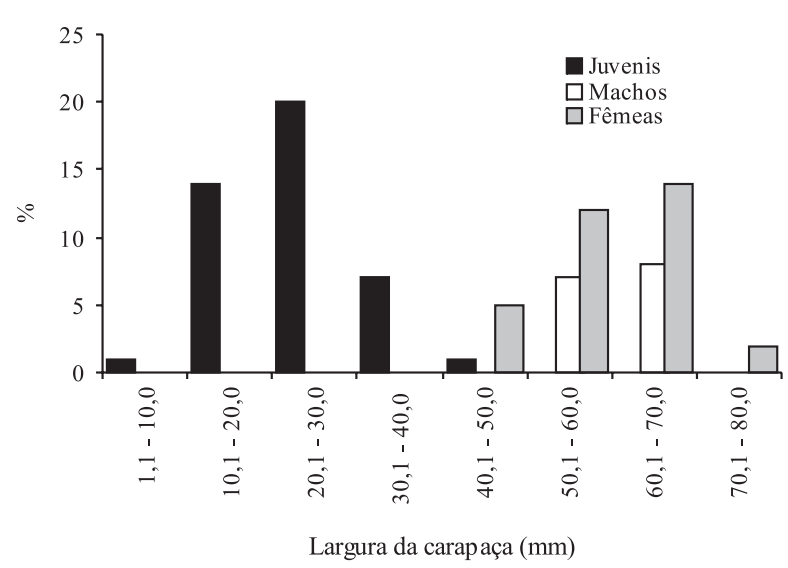

Fig. 1. Distribuição de machos, fêmeas e juvenis de Ucides cordatus (Linnaeus, 1763) por classes de tamanho, durante o período de outubro de 2000 a março de 2002, obtidos no manguezal de Itacuruçá - Coroa Grande, Estado do Rio de Janeiro. 
na taxa de sobrevivência ao longo dos seis meses seguintes, voltando a reduzir nos meses finais de observação (Fig. 2). A alta mortalidade observada durante o período de confinamento provavelmente ocorreu devido às dificuldades durante a aquisição de cálcio e suplementos orgânicos para o endurecimento do novo tegumento (ADIYODI \& ADIYODI, 1970), ou devido à quantidade insuficiente de alimento ou nutrientes na dieta do caranguejo durante o experimento (HARTNOLL, 1982).

OSTRENSKY et al. (1995) obtiveram durante 90 dias uma sobrevivência de $76 \%$ para exemplares de $U$. cordatus mantidos em diferentes ambientes, no laboratório e em cercos no manguezal. De acordo com esses autores, a mortalidade observada durante o estudo pode ter ocorrido devido à utilização de diferentes itens alimentares durante todo o experimento - folhas de mangue, alguns vegetais e peixes - lutas e até canibalismo durante o processo de muda. No presente estudo, em nenhum momento foi verificado canibalismo nos tanques de estudo, provavelmente devido à grande disponibilidade de abrigos compostos por pedaços de tubos de PVC, que serviram de esconderijos para os exemplares em confinamento, e à elevada quantidade de alimento fornecido.

Do total de caranguejos observados (15 machos, 33 fêmeas e 43 juvenis), 43 exemplares (47\%) - 5 machos, 14 fêmeas e 24 juvenis - realizaram pelo menos uma muda em cativeiro. Os juvenis fizeram duas mudas durante os oito meses, enquanto os adultos realizaram até três mudas num período de mais dez meses. Apesar do curto período em laboratório, os caranguejos juvenis cresceram numa taxa maior que os animais adultos, provavelmente para desempenhar em curto prazo um importante papel no estoque reprodutivo da população ao atingirem a maturidade sexual (HARTNOLL, 1982; SASTRY, 1983). De acordo com LóPEz \& RoDRíGUEz (1998), Chasmagnathus granulata Dana, 1851 realiza mais de quatro mudas consecutivas, sendo mais freqüente três mudas no máximo, para atingir a maturidade sexual.

Geraldes \& CALventi (1983) observaram que os exemplares menores de $U$. cordatus mantidos em condições de cativeiro podem mudar de carapaça até três vezes num período de seis meses, enquanto os animais

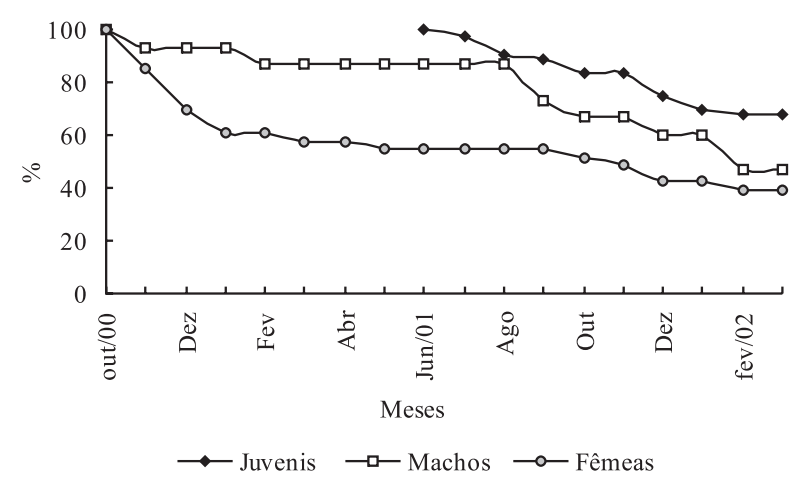

Fig. 2. Sobrevivência mensal de Ucides cordatus (Linnaeus, 1763) em laboratório, durante o período de outubro de 2000 a março de 2002. maiores mudam apenas uma vez ao ano. Provavelmente a ocorrência de mudas sucessivas nos exemplares mantidos em laboratório pode ter sido favorecida pela disponibilidade e fluxo contínuo de água nos tanques de observação. De acordo com HarTnOll (1988), a disponibilidade de água é mais importante do que a temperatura no processo de muda em caranguejos semiterrestres, uma vez que há o risco de dessecação após a muda.

Ucides cordatus apresentou um padrão sazonal para realização do processo de muda, sugerindo uma preferência por meses mais quentes, principalmente naqueles correspondentes a primavera e verão, observando pico durante o mês de novembro. Durante os meses de baixa temperatura (maio, junho e julho), não foi observada a ocorrência de muda em cativeiro (Fig. 3). Os resultados obtidos corroboram as observações de PINHEIRO \& FisCARELLI (2001), que mencionaram maior ocorrência de muda para a espécie durante os meses da primavera. Em todo o estudo, verificou-se que 88,3\% das mudas foram realizadas ao longo do período matutino, sendo que $11,7 \%$ observadas entre os juvenis durante o período noturno. Estes resultados discordam das observações de Broekhuysen (1941), HyATt (1948), GERALdes \& CALVENTi (1983) e LóPEz \& RodríGuez (1998), que estudaram, respectivamente, Cyclograpsus punctatus H. Milne-Edwards, 1837, Pachygrapsus crassipes Randall, 1839, U. cordatus e C. granulata. Tais trabalhos verificaram a ocorrência do processo de muda durante o período noturno, provavelmente para minimizar o efeito do canibalismo sobre indivíduos.

Por outro lado, a ausência de muda em temperaturas mais baixas é um processo conhecido entre os braquiúros (Hyatt, 1948; Boschi et al., 1967; Tsuchida \& Watanabe, 1997). BRoEKhUYSEN (1941) relata, para C. punctatus observados na África do Sul, que a realização de mudas é rara entre os meses de maio e agosto, quando a temperatura é mais baixa. Em muitos crustáceos a freqüência de muda é influenciada por fatores sazonais, sendo que entre os organismos aquáticos a variação da temperatura é o principal fator estimulador de mudas (HARTNOLL, 1982).

Ao contrário do estudo de AdIYODI (1988), a muda é programada normalmente entre os machos a poucas

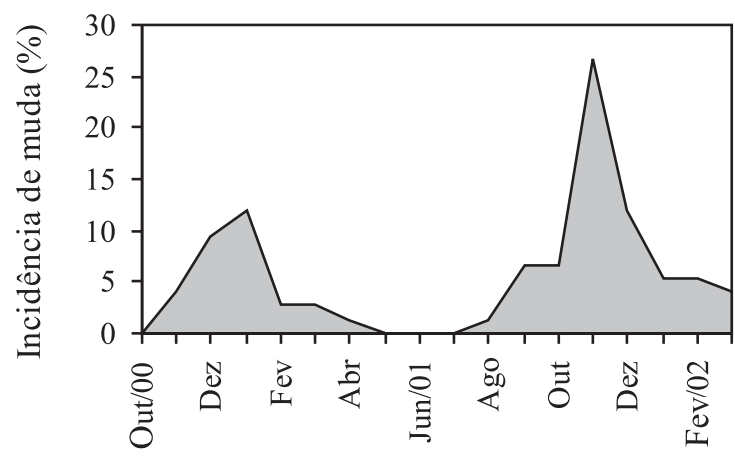

Meses

Fig. 3. Freqüência de muda do caranguejo Ucides cordatus (Linnaeus, 1763) durante o período de outubro de 2000 a março de 2002. 
semanas antes do processo de muda das fêmeas (Fig. 4). Este fato, provavelmente, não foi observado claramente devido ao dobro de exemplares fêmeas obtidos em relação aos indivíduos machos. Segundo Hartnoll (1969), a cópula entre caranguejos geralmente envolve machos em estágio de intermuda e fêmeas em estágios de muda recente, sendo que os machos devem estar sexualmente maduros para garantir a transferência dos espermatóforos aos gonóporos das fêmeas (HARTNOLL, 1978; GoNZÁLEZGURRIARÁN \& FREIRE, 1985).

O percentual médio do incremento da carapaça foi de $2,09 \pm 1,8 \%$, verificando-se uma média de $2,9 \pm 2,1 \%$ entre os juvenis, $2,2 \pm 1,4 \%$ entre os machos e $1,3 \pm 0,8 \%$ entre as fêmeas. Verificou-se, todavia, que o incremento de muda observado para $U$. cordatus apresentou diferença significativa $(\mathrm{p}<0,05)$ quando se compararam machos e fêmeas, bem como adultos e juvenis (Tab. I).

Dentre as espécies estudadas até então, o sexo parece não influenciar o percentual de incremento de muda, como em C. maenas (CROTHERs, 1967), Rhithropanopeus harrisii (Gould, 1841) (TUROBOYsкI, 1973), C. punctatus (BroeKhuYsen, 1941) e G. cruentata (Silva \& Oshiro, 2002). No entanto, o menor tamanho percentual em relação ao incremento de muda verificado para as fêmeas pode ser atribuído a uma reduzida longevidade desses indivíduos (HenNing, 1975), ou ainda devido ao menor crescimento somático atingido depois que o animal alcança a maturidade sexual (HARTNOLL, 1982).

Tabela I. Crescimento do caranguejo Ucides cordatus (Linnaeus, 1763) em laboratório. Adultos mantidos durante dezoito meses e juvenis, durante oito meses em laboratório.

\begin{tabular}{lccc}
\hline Parâmetros & Machos & Fêmeas & Juvenis \\
\hline Total de animais & 15 & 33 & 43 \\
Número de muda & 18 & 34 & 80 \\
$\begin{array}{l}\text { Número máximo de muda } \\
\text { Variação do incremento }\end{array}$ & 0,8 a 4,2 & 0,3 a 2,9 & 0,2 a 7,5 \\
$\begin{array}{l}\text { de muda (mm) } \\
\begin{array}{l}\text { Média do incremento } \\
\text { de muda (\%) }\end{array}\end{array}$ & $2,2 \pm 1,4$ & $1,3 \pm 0,8$ & $2,9 \pm 2,1$ \\
$\begin{array}{l}\text { Média do período de } \\
\text { intermuda (dias) }\end{array}$ & $191 \pm 140$ & $216 \pm 76,2$ & $54 \pm 1,4$ \\
$\begin{array}{l}\text { Variação do período de } \\
\text { intermuda (dias) }\end{array}$ & 65 a 408 & 37 a 406 & 22 a 227
\end{tabular}

intermuda (dias)

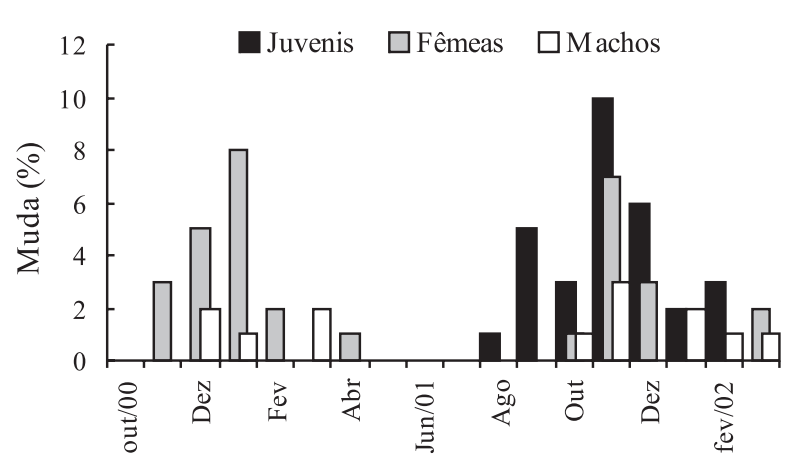

Meses

Fig. 4. Freqüência de muda em machos, fêmeas e juvenis de Ucides cordatus (Linnaeus, 1763) durante o período de outubro de 2000 a março de 2002.
OSTRENSKY et al. (1995) observaram um incremento médio de muda de $2,5 \%$ quando foi fornecida uma dieta à base de folhas de mangue aos indivíduos confinados, sendo este percentual similar ao obtido neste estudo. De acordo com MAuCHLINE (1976), o incremento de muda não depende somente da alimentação, mas da idade e do tamanho do exemplar analisado. Segundo HenNING (1975), C. guanhumi apresenta uma redução no incremento de muda de acordo com o tamanho do exemplar observado, sendo o incremento em torno de $10 \%$ para animais com largura da carapaça de $10 \mathrm{~mm}$ e de $5 \%$ para aqueles cuja largura excede $50 \mathrm{~mm}$. Já para o grapsídeo Aratus pisonii (H. Milne-Edwards, 1837), o percentual de incremento para animais de $12 \mathrm{~mm}$ de largura encontra-se na faixa de $10 \%$, enquanto os exemplares maiores crescem apenas 3\% (WARNER, 1967).

A relação entre o incremento de muda (IM) e a largura da carapaça (LC) apresentou uma correlação negativa, diminuindo com o tamanho do animal (Fig. 5), podendo ser expressa pela equação: $\mathrm{IM}=-0,0707 \mathrm{LC}+$ $4,645(r=0,40)$. Essa diminuição no incremento de muda é comum entre os braquiúros, sendo igualmente observada em outras espécies como: P. crassipes, Hemigrapsus oregonensis (Dana, 1851) e H. nudus (Dana, 1851) por Olmsted \& BAumberger (1923), A. pisonii por Warner (1967) e G. cruentata por SILVA \& OsHIRo (2002).

PinheIro \& FisCARELli (2001) mencionam que $U$. cordatus tem apresentado uma extraordinária redução no crescimento, apesar do elevado tamanho apresentado pelos animais quando adultos. Em indivíduos mais jovens, a taxa de crescimento é mais rápida e diminui progressivamente com o alcance da maturidade sexual (TRAVIS, 1954; HancoCK \& Edwards, 1967; HaRTNOLl, 1982).

A média do período de intermuda nos machos foi de $191 \pm 140$ dias ( 65 a 408 dias), $216 \pm 76,2$ dias ( 37 a 406 dias) entre as fêmeas e $54 \pm 1,41$ dias ( 22 a 227 dias) entre os juvenis, verificando-se uma diferença significativa entre os valores médios entre os indivíduos analisados $(\mathrm{p}<0,05)$ (Tab. I).

BOND \& BUCKUP (1988), em estudos com Macrobrachium borelli (Nobili, 1896), bem como SiLvA \& Oshiro (2002), com G. cruentata, constataram que o tamanho do animal interfere na duração do período de intermuda, observando-se um período menor entre os

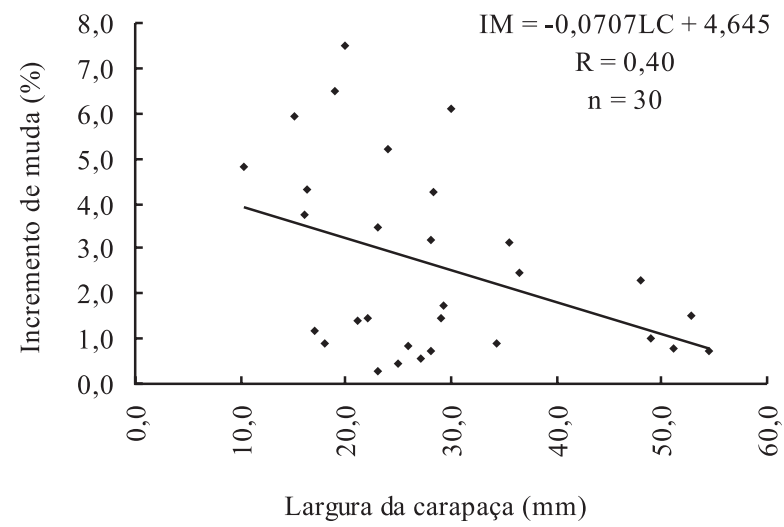

Fig. 5. Relação entre o incremento de muda (IM) e a largura da carapaça (LC) de Ucides cordatus (Linnaeus, 1763) durante o período de outubro de 2000 a março de 2002. 
juvenis do que entre os animais de maior porte.

De acordo com ChitTLEBOROUGH (1975), outro fator que pode influenciar na duração do período de intermuda é a redução quali-quantitativa do alimento fornecido e ingerido pelos animais analisados. Durante observações com relação ao fornecimento de alimentos em Panulirus longipes (H. Milne-Edwards, 1868) em cativeiro, o autor verificou a ocorrência de muda em torno de 42 dias, quando manteve um fornecimento diário. No entanto, quando o alimento passou a ser fornecido durante duas vezes e/ou uma vez por semana, o crescimento somático observado passou a ocorrer num período maior de tempo, entre 51 e 79 dias, respectivamente.

Os resultados obtidos em laboratório para o caranguejo-uçá mostraram que o confinamento parece não inibir o crescimento, embora tenha ocorrido um reduzido crescimento somático ao longo de duas ou mais mudas sucessivas. A espécie apresentou crescimento lento, necessitando de maiores esforços para o manejo das populações naturais, uma vez que a manutenção em cativeiro é impraticável dado ao baixo incremento de muda e longo período para a ocorrência de mudas sucessivas.

Agradecimentos. À Fundação de Amparo à Pesquisa no Estado do Rio de Janeiro (Processo E-26/172.013/1999), ao Sr. Casemiro Antônio Alves, pelo auxílio nas coletas de campo e nas atividades diárias e a Zilanda de Souza Silva, pela assistência durante a organização dos dados e alimentação dos animais.

\section{REFERÊNCIAS BIBLIOGRÁFICAS}

AdiYodi, R. G. 1988. Reproduction and development. In: BURGGREN, W. W. \& Mсманоn, B. R. eds. Biology of the land crabs. New York, Cambridge University Press. p.139-185.

Adiyodi, K. G. \& AdiYodi, R. G. 1970. Endocrine control of reproduction in Decapoda Crustacea. Biological Review 45:121-165.

Aiken, D. E. 1973. Proecdysis, setal development, and molt prediction in the american lobster (Homarus americanus) Journal of the Fishery Research Board of Canada 30: $1337-1344$.

AlCÂNTARa Filho, P. 1978. Contribuição para o conhecimento da biologia e ecologia do caranguejo uçá, Ucides cordatus (Linnaeus, 1763) (Crustacea, Decapoda, Brachyura) no manguezal do rio Ceará (Brasil). Arquivos de Ciências do Mar 18:1-41.

Blankensteyn, A.; Cunha Filho, D. \& Freire, A. S. 1995. Distribuição, estoques pesqueiros e conteúdo protéico do caranguejo do mangue Ucides cordatus (L., 1763) (Brachyura, Ocypodidae) nos manguezais da baía das Laranjeiras e adjacências, Paraná, Brasil. Arquivos de Biologia e Tecnologia 40(2):331-349.

Bond, G. \& Buckup, L. 1988. O ciclo da intermuda em Macrobrachium borelii (Nobili, 1896) (Crustacea, Decapoda, Palaemonidae): a influência da temperatura e do comprimento do animal. Revista Brasileira de Zoologia 5(1):45-59.

Boschi, E.; Scelzo, M. \& Goldstein, B. 1967. Desarrollo larval de dos especies de crustáceos decápodos en el laboratorio: Pachycheles haigae Rodríguez da Costa (Porcellanidae) y Chasmagnathus granulata Dana (Grapsidae). Boletin del Centro de Investigaciones Biológicas Mar del Plata 12:1-46.

Branco, J. O. 1993. Aspectos bioecológicos do caranguejo uçá, Ucides cordatus (Linnaeus, 1763) (Crustacea, Decapoda) do manguezal do Itacorubi, Santa Catarina, Brasil. Arquivos de Biologia e Tecnologia 36(1):133-148.

BroekhuYsen, G. J. 1941. The life history of Cyclograpsus punctatus, M. Edw.: breeding and growth. Transaction of the Royal Society of South Africa 28:331-366.

Castro, A. C. L. 1986. Aspectos bio-ecológicos do caranguejouçá, Ucides cordatus cordatus (Linnaeus 1763), no estuário do rio dos cachorros e estreito do coqueiro, São Luís - MA Boletim do Laboratório de Hidrobiologia 7:7-26.

Chittleborough, R. G. 1975. Environmental factors affecting growth and survival of juvenile western rock lobsters Panulirus longipes ( $\mathrm{H}$. Milne-Edwards). Australian Journal of Marine and Freshwater Research 26:177-196.

Costa, R. S. 1979. Bioecologia do caranguejo uçá, Ucides cordatus (Linnaeus, 1763) - Crustacea, Decapoda no nordeste brasileiro. Boletim Cearense de Agronomia 20:1-74.

Crothers, J. H. 1967. The biology of the shore crab, Carcinus maenas (L.) 1. The background-anatomy, growth and life history. Field Studies 2:407-434.

Dalabona, G.; Loyola e Silva, J. \& Pinheiro, M. A. A. 2005. Size at morphological maturity of Ucides cordatus (Linnaeus, 1763) (Brachyura, Ocypodidae) in the Laranjeiras Bay, Southern Brazil. Brazilian Archives of Biology and Technology 48(1): 139-145.

GonzÁlez-Gurriarán, E. \& Freire, J. 1985. Reproducción de la nécora Macropipus puber (L.) (Decapoda, Brachyura), y ciclo reproductivo en la Ría de Arousa (Galicia, NW España). Boletin del Instituto Español del Oceanografia 2(1):10-32.

Geraldes, M. G. \& Calventi, I. B. 1983. Estudios experimentales para el mantenimiento en cautiverio del cangrejo Ucides cordatus. Ciencia Interamericana 23(1-4):41-53.

HancoCK, D. A. \& Edwards, E. 1967. Estimation of animal growth in the edible crab (Cancer pagurus L.). Journal du Conseil International pour I'Exploration de la Mer 31(2):246-264.

Harris, R. R. \& Santos, M. C. F. 2000. Heavy metal contamination and physiological variability in the brazilian mangrove crabs Ucides cordatus and Callinectes danae (Crustacea: Decapoda). Marine Biology 137:691-703.

Hartnoll, R. G. 1969. Mating in Brachyura. Crustaceana 16:161-181.

1978. The determination of relative growth in Crustacea Crustaceana 34(3):281-293.

. 1982. Growth. In: Buiss, D. E. ed. The biology of Crustacea embriology, morphology and genetics. New York, Academic Press. p.111-196

1988. Growth and molting. In: BURGGREN, W. W. \& MCMAHON, B. R. eds. Biology of the land crabs. New York, Cambridge University. p.186-210.

Hattori, G. Y. \& Pinheiro, M. A. A. 2003. Fertilidade do caranguejo de mangue Ucides cordatus (Linnaeus) (Crustacea, Brachyura, Ocypodidae), em Iguape (São Paulo, Brasil). Revista Brasileira de Zoologia 20(2):309-313.

Henning, H. G. 1975. Ökologische, ethologische und sinnesphysiologische untersuchungen an der landkrabbe Cardisoma guanhumi Latreille (Decapoda, Brachyura). Forma et Functio 8:253-304

Hepper, B. T. 1967. On the growth at moulting of lobsters (Homarus vulgaris) in Cornwall and Yorkshire. Journal of the Marine Biological Association of the United Kington 47:629-643.

Hewett, C. J. 1974. Growth and moulting in the common lobster (Homarus vulgaris Milne-Edwards). Journal of the Marine Biological Association of the United Kington 54:379-391.

Hyatт, R. 1948. The biology of the lined shore crab Pachygrapsus crassipes Randall. Pacific Sciencie 2:135-213.

Ivo, C. T. C. \& Vasconcelos, E. M. S. 2000. Potencial reprodutivo do caranguejo-uçá, Ucides cordatus cordatus (Linnaeus, 1763) capturado na região estuarina do Rio Curimatau (Canguaretama, Rio Grande do Norte, Brasil). Boletim Técnico Científico CEPENE 8(1):7-14

Lobão, V. L.; Roverso, E. A.; Lage, M. \& Hortencio, E. 1996. Ciclo de muda e crescimento em Macrobrachium amazonicum Heller, 1862 e Macrobrachium rosenbergii De Man (Decapoda, Palaemonidae). Boletim do Instituto de Pesca 23:35-45.

López, L. S. \& Rodríguez, E. M. 1998. Somatic growth of the estuarine crab Chasmagnathus granulata Dana, 1851 (Brachyura, Grapsidae), under laboratory conditions. Investigaciones Marinas 26:127-135.

MaiA, A. Z. \& Penna, T. M. P. A. 1982. Ecologia dos crustáceos decápodos do mangue de Guaratiba. Rio de Janeiro, 
FEEMA, Departamento de Conservação Ambiental. 96p.

Mauchline, J. 1976. The Hiatt growth diagram for Crustacea. Marine Biology 35:79-84.

Melo, G. A. S. 1996. Manual de identificação dos Brachyura (caranguejos e siris) do litoral brasileiro. São Paulo, Plêiade/FAPESP. 604p.

Mota Alves, M. I. 1975. Sobre a reprodução do caranguejo uçá, Ucides cordatus (Linnaeus, 1763) em mangues do Ceará (Brasil). Arquivos de Ciências do Mar 15(2):85-91.

Mota Alves, M. I. \& Madeira, P. H., JR. 1980. Algumas considerações sobre a respiração do caranguejo uçá Ucides cordatus (Linnaeus, 1763) (Crustacea, Decapoda). Arquivos de Ciências do Mar 20(1/2):63-69.

NoRdi, N. A. 1994. A captura do caranguejo-uçá (Ucides cordatus) durante o evento reprodutivo da espécie: o ponto de vista dos caranguejeiros. Revista Nordestina de Biologia 9(1):41-47.

Oliveira, L. P. H. 1946. Estudos biológicos e ecológicos dos crustáceos comestíveis uçá e guaiamum, Ucides cordatus (Linnaeus) e Cardisoma guanhumi (Latreille) Brachyura, Gecarcinidae. Memórias do Instituto Oswaldo Cruz 44(2):295-322.

Olmsted, J. M. D. \& Baumberger, J. P. 1923. Form and growth of grapsoid crabs. A comparison of the form of three species of grapsoid crabs and their growth at molting. Journal of Morphology 38:279-294.

Ostrensky, A.; Sternhain, U. S.; Brun, E.; Wegbecher, F. X. \& Pestana, D. 1995. Análise da viabilidade técnico-econômica dos cultivos do caranguejo-uçá Ucides cordatus (Linnaeus, 1763) no litoral paranaense. Arquivos de Biologia e Tecnologia 38(3):939-947.

Pinheiro, M. A. A. \& Fiscarelli, A. G. 2001. Manual de apoio à fiscalização - caranguejo-uçá (Ucides cordatus). Itajaí, UNESP / CEPSUL / IBAMA. 43p.

Pinheiro, M. A. A.; Baveloni, M. D. \& Terceiro, O. S. L. 2003. Fecundity of the mangrove crab Ucides cordatus (Linnaeus, 1763) (Brachyura, Ocypodidae). Invertebrate Reproduction and Development 43(1):19-26.

Pinheiro, M. A. A.; Fiscarelli, A. G. \& Hattori, G. Y. 2005. Growth of the mangrove crab Ucides cordatus (Brachyura, Ocypodidae). Journal of Crustacean Biology 25(2):293-301.

Reid, D. G.; Abello, P.; Kaiser, M. J. \& Warman, C. G. 1997. Carapace colour, inter-moult duration and the behavioral and physiological ecology of the shore crab Carcinus maenas. Estuarine, Coastal and Shelf Science 44:203-211.
Rodrigues, A. M. T.; Branco, E. J.; Saccardo, S. A. \& Blankensteyn, A. 2000. A explotação do caranguejo Ucides cordatus (Decapoda:Ocypodidae) e o processo de gestão participativa para normatização da atividade na região Sudeste-Sul do Brasil. Boletim do Instituto de Pesca 26(1):63-78.

Rodrigues, M. D. \& Hebling, N. J. 1989. Ucides cordatus cordatus (Linnaeus, 1763) (Crustacea, Decapoda). Complete larval development under laboratory conditions and its systematic position. Revista Brasileira de Zoologia 6(1):147-166.

SARDÁ, F. 1983. Determinación de los estados de intermuda en Nephrops norvegicus (L.), mediante la observación de los pleópodos. Investigaciones Pesqueras 47(1):95-112.

. 1985. Estudio de la edad, crecimiento y frequencia de muda, en cautividad, de Nephrops norvegicus (L.) durante las etapas del ciclo de intermuda. Investigaciones Pesqueras 49(2): $139-154$

1991. Reproduction and moult synchronism in Nephrops norvegicus (L.) (Decapoda, Nephropidae) in the Western Mediterranean: is spawning annual or biennual? Crustaceana 60(2): $186-199$.

SASTRY, A. 1983. Ecological aspects of reproduction. In: Bliss, D. E. ed. The biology of Crustacea. New York, Academic Press. p.179-269.

Silva, Z. \& Oshiro, L. M. Y. 2002. Crescimento em Goniopsis cruentata (Latreille) (Crustacea, Brachyura, Grapsidae) em laboratório. Revista Brasileira de Zoologia 19(3):915-923.

Sмiтh, D. L. 1990. Patterns of the limb loss in the blue crab, Callinectes sapidus Rathbun, and the effects of autotomy on growth. Bulletin of Marine Science 46(1):23-36.

Thomas, H. J. 1958. Observations on the increase in size at moulting in the lobster (Homarus vulgaris M.-Edw.). Journal of the Marine Biological Association of the United Kingdon 37:603-606.

Travis, D. F. 1954. The moulting cycle of the spiny lobster, Panulirus argus Latreille. I. Molting and growth in laboratorymaintained individuals. Biological Bulletin 107(3):433-450.

Tsuchida, S. \& Watanabe, S. 1997. Growth and reproduction of the grapsid crab Plagusia dentipes (Decapoda: Brachyura). Journal of Crustacean Biology 17(1):90-97.

TURoвоYsky, K. 1973. Biology and ecology of the crab Rhithropanopeus harrisii ssp. tridentatus. Marine Biology 23:303-313.

WARNER, G. F. 1967. The life history of the mangrove tree crab, Aratus pisonii. Journal of Zoology 153:321-325.

Recebido em abril de 2005. Aceito em agosto de 2006. ISSN 0073-4721

Artigo disponível em: www.scielo.br/isz 